\title{
Limiting Stable Currents in Bounded Electron and Ion Streams
}

\author{
W. B. BRIDGES, Member, IeEe, J. I. FREY, AND C. K. BIRDSALl, fellow, Iege
}

\begin{abstract}
The classical static analysis of the infinite planar diode has been extended to include the effects of finite transverse beam size. Simple expressions have been found for the increase in maximum stable current density over that of an infinite stream for finite cylindrical and strip streams flowing between plates of infinite diodes. The results are also given in terms of stream perveance. The effect of a nonuniform distribution of current across the stream is shown to be relatively small. Experimental values of maximum stable current agree with those obtained from the analysis. A further extension of the static analysis has been made to include the effects of additional conducting plane boundaries parallel to the stream motion. For length-to-width ratios $L / D$ less than 0.25 the tube is adequately described by the results for the infinite planar aiode and for L/D greater than 4, the infinitely-long drift tube theory suffices. At intermediate values of $L / D$, the maximum amount of current that can be stably passed through the tube is greater than that predicted by either asymptotic theory.
\end{abstract}

\section{INTRODUCTION}

$\mathbb{V}$ ALUES OF limiting current are given for the finite diameter stream in a diode in Section $I$ and for a finite-length planar drift tube in Section II. These time-independent solutions complement the well-known solutions for infinitely broad diodes [1]-[3] and infinitely long drift tubes [4]-[8]. The fields (or potentials) in the infinite diode and the infinite drift tube vary along only one coordinate; the fields in the present models vary both radially and axially. The motion of the electron or ion stream, however, is constrained to the axial direction by a strong axial magnetic field, so that the current density is constant along the direction of motion.

Limiting current in this time-independent type of analysis is established in a special way. Solutions with unidirectional flow for which $V(\mathbf{r})>0$ are found for currents increasing from zero. Such solutions cannot be found beyond a certain value of current, and this value is called the limiting current. Energy relations and small-signal stability at this "limiting" value have been discussed by Bridges and Birdsall [9]. Beyond this value of current, only solutions with bidirectional flow are expected. A timedependent solution that would show time growth leading to large amplitude oscillations beyond limiting current [9] is only implied and is not presented here.

In Section I, several finite-diameter stream cross sections in a diode are analyzed, and the results checked

Manuscript received January 25, 1965. The work was supported in part by the Joint Services Electronics Program under Grant No. AF-AFOSR-139-64.

W. B. Bridges is with Hughes Research Labs., Malibu, Calif

J. I. Frey and C. K. Birdsall are with Electronics Research Lab., Dept. of Electrical Engineering, University of California, Berkeley, Calif. against experiment. In Section II, the effect of adding side walls (making a planar drift tube with ends) is found and the results related to both the diode and infinitedrift-tube solutions.

\section{Finite Diameter Stream in an INFINITE Dione ${ }^{1}$}

The model for this analysis is a cylindrical stream flowing normal to the electrodes shown in Fig. 1. A variety of radial distributions of current density, including hollow streams and streams with no definite boundary, is allowed; in the latter, $b$ is to be interpreted as a "characteristic" radius. In the configuration shown, electric fields exist outside as well as inside the stream. Some of the field lines from charges located near the center of the region $(z=a / 2)$ terminate on the electrodes at points outside the stream; charges located near an electrode thus "see" less electric field produced by charges near the center than if the stream were infinitely broad. ${ }^{2}$ A given current density thus produces less space-charge depression of potential, and the current density necessary to produce limiting is thereby increased.

\section{A. Method of Solution}

The problem is to integrate Poisson's equation for the potential $V(r, z)$ in two dimensions;

$$
\nabla^{2} V(r, z)=-\rho(r, z) / \epsilon_{0} .
$$

The charge density $\rho(r, z)$ is related to the current and velocity by

$$
i(r, z)=\rho(r, z) v(r, z) .
$$

We use the time-independent, zero-temperature equation of motion for a single-valued velocity,

$$
\frac{1}{2} m v^{2}=-e V \text {. }
$$

In the infinitely broad diode or the infinitely long drift tube, potential depends on only one coordinate, and closed-form solutions can be obtained. With two-coordinate dependence, no closed-form solutions have been found. An approximate solution using a Fourier-Bessel expansion is given in this Section for the diode; a second method is used in Section II with the drift tube.

${ }^{1}$ Section I comprises a portion of W. B. Bridges' Ph.D. dissertation [14].

2 This is analogous to the situation arising with the Hahn-Ramo space-charge waves. As a very broad stream is reduced to a thin stream, a given bunching of charge produces a smaller and smaller axial electric field. This effect is accounted for in the space-charge reduction factors, as tabulated by Branch and Mihran [10]. 


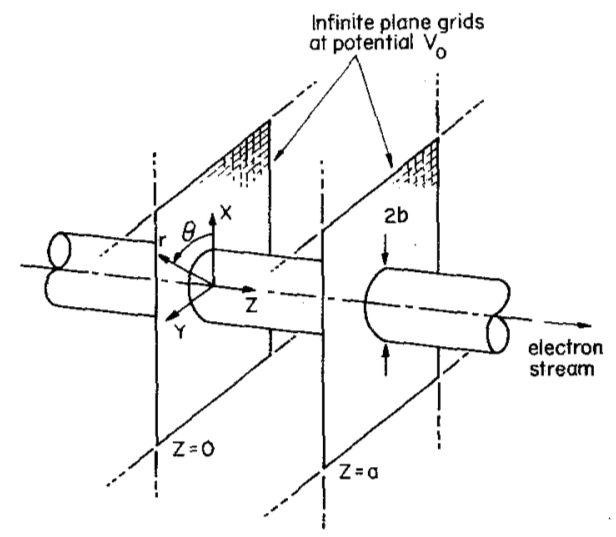

Fig. 1. Stream and electrode configuration for the finite cylindrical stream model.

For the diode, we write the potential $V(r, z)$ and the charge density $\rho(r, z)$ as expansions in suitable functions. The current density $i$ is assumed to be independent of $z$, for example, due to a strong axial magnetic field; several simple forms are later assumed for the radial distribution of the current. The potential is written as

$$
V(r, z)=\dot{V}_{0}+\sum_{m=1}^{\infty} \int_{0}^{\infty} F_{m}(k) \sin \left(\frac{m \pi z}{a}\right) J_{0}(k r) k d k
$$

where the expansion coefficients are given by

$F_{m}(k)=\frac{2}{a} \int_{0}^{a} \int_{0}^{\infty} V\left(r^{\prime}, z^{\prime}\right) \sin \left(\frac{m \pi z^{\prime}}{a}\right) J_{0}\left(k r^{\prime}\right) r^{\prime} d r^{\prime} d z^{\prime}$.

The charge density is expanded in a similar series

$$
\rho(r, z)=\sum_{m=1}^{\infty} \int_{0}^{\infty} P_{m}(k) \sin \left(\frac{m \pi z}{a}\right) J_{0}(k r) k d k,
$$

where

$P_{m}(k)=\frac{2}{a} \int_{0}^{a} \int_{0}^{\infty} \rho\left(r^{\prime}, z^{\prime}\right) \sin \left(\frac{m \pi z^{\prime}}{a}\right) J_{0}\left(k r^{\prime}\right) r^{\prime} d r^{\prime} d z^{\prime}$.

Substitution of these expressions into Poisson's equation yields the relations between the expansion coefficients,

$$
F_{m}(k)\left[\left(\frac{m_{\pi}}{a}\right)^{2}+k^{2}\right]=\frac{1}{\epsilon_{0}} P_{m}(k)
$$

The potential can then be written in terms of the charge density as

$$
\begin{aligned}
V(r, z) & =V_{0}+\sum_{m=1}^{\infty} \int_{0}^{\infty}\left[\frac{2}{a \epsilon_{0}} \int_{0}^{a} \int_{0}^{\infty} \rho\left(r^{\prime}, z^{\prime}\right)\right. \\
& \left.\sin \left(\frac{m \pi z^{\prime}}{a}\right) J_{0}\left(k r^{\prime}\right) r^{\prime} d r^{\prime} d z^{\prime}\right] \\
& \cdot\left\{\left(\frac{m \pi}{a}\right)^{2}+k^{2}\right\}^{-1} J_{0}(k r) k d k \sin \left(\frac{m \pi z}{a}\right) .
\end{aligned}
$$

The second equation that the potential and charge density must satisfy is (2). Equations (2) and (9) can be solved numerically, given the transverse distribution $i(r)$. This distribution is chosen so as to approximate the current density variations occurring in typical electron devices.
A normalized current distribution $\Psi_{r}(r)$ is introduced;

$$
i(r)=\rho(r, 0) v_{0}=\rho_{0} v_{0} \Psi_{r}(r)=i_{0} \Psi_{r}(r) .
$$

For a rectangular distribution $i_{0}$ is related to the total current $I_{0}$ by $I_{0}=\pi b^{2} i_{0}$. The charge density $\rho(r, z)$ is assumed to be separable;

$$
\rho(r, z)=\rho_{0} \Psi_{r}(r) \cdot \Psi_{z}(z),
$$

where $\Psi_{z}(z)$ is the normalized longitudinal distribution of charge density. Clearly, $\Psi_{z}(0)=1$. Equation (8) thus becomes

$V(\sigma, \zeta)=V_{0}+2 \frac{\rho_{0} a^{2}}{\epsilon_{0}}$

$\cdot \sum_{m=1}^{\infty}\left[\left(\beta_{r}^{2} \int_{0}^{\infty} \int_{0}^{\infty} \frac{\Psi_{r}\left(\sigma^{\prime}\right) J_{0}\left(\gamma_{r} \sigma^{\prime}\right) J_{0}\left(\gamma_{r} \sigma\right)}{\beta_{r}^{2}+\gamma_{r}^{2}} \sigma^{\prime} \gamma_{r} d \sigma^{\prime} d \gamma_{r}\right)\right.$

$\left.\cdot\left(\frac{1}{(m \pi)^{2}} \int_{0}^{1} \Psi_{z}\left(\zeta^{\prime}\right) \sin \left(m \pi \zeta^{\prime}\right) d \zeta^{\prime}\right)\right] \sin (m \pi \zeta)$,

where the normalized constants and variables

$$
\begin{aligned}
\zeta & =z / a, \\
\sigma & =r / b, \\
\beta_{r} & =\frac{m \pi b}{a}, \\
\gamma_{r} & =k b,
\end{aligned}
$$

have been introduced.

For a very broad stream $(b \rightarrow \infty)$ the potential is given by

$V(\zeta)=V_{0}+\frac{2 \rho_{0} a^{2}}{\epsilon_{0}} \sum_{m=1}^{\infty}\left[\frac{1}{(m \pi)^{2}} \int_{0}^{1} \Psi_{z}\left(\zeta^{\prime}\right) \sin \left(m \pi \zeta^{\prime}\right) d \zeta^{\prime}\right]$ $\cdot \sin (m \pi \zeta)$

This form differs from (12) by the reduction factor,

$$
\begin{aligned}
\beta_{r}^{2} \int_{0}^{\infty} \int_{0}^{\infty} \frac{\Psi_{r}\left(\sigma^{\prime}\right) J_{0}\left(\gamma_{r} \sigma^{\prime}\right) J_{0}\left(\gamma_{r} \sigma\right)}{\beta_{r}^{2}+\gamma_{r}^{2}} \sigma^{\prime} d \sigma^{\prime} \gamma_{r} d \gamma_{r} \\
\\
\equiv R_{m}\left(\beta_{r}, \sigma\right) .
\end{aligned}
$$

This factor is evaluated below for simple current distributions.

\section{B. Results of Analysis}

1) Uniform Current, Solid Stream: For a uniform eurrent distribution

$$
\Psi_{\tau}(\sigma)=\left\{\begin{array}{ll}
1, & |\sigma| \leq 1 \\
0, & |\sigma|>1
\end{array},\right.
$$

and the reduction factor becomes

$$
\begin{aligned}
R_{m}\left(\beta_{r}, \sigma\right) & =\beta_{r}^{2} \int_{0}^{\infty} \frac{J_{0}\left(\gamma_{r} \sigma\right)}{\beta^{2}+\gamma_{r}^{2}} \int_{0}^{1} J_{0}\left(\gamma_{r} \sigma^{\prime}\right) \sigma^{\prime} d \sigma^{\prime} \gamma_{r} d \gamma_{r} \\
& =1-\beta_{r} I_{0}\left(\beta_{r} \sigma\right) K_{1}\left(\beta_{r}\right) .
\end{aligned}
$$

For a thin stream, $I_{0}\left(\beta_{r} \sigma\right) \cong 1$ in the first few terms of the series; for a thicker stream this approximation is not 
so good, but $\beta_{r} K_{1}\left(\beta_{r}\right)$ is small, and the error introduced by taking $I_{0}\left(\beta_{r} \sigma\right) \cong 1$ is still small. Physically, this means that the potential varies only a little over the stream cross section. At this point we take the potential to be the value on the axis, $\sigma=0$. Some average over the stream cross section could be made in this simple case, but this refinement is probably not justified considering the other approximations involved. All $r$-dependence is thus removed, and one-dimensional equations are regained; the effects of the transverse variations are included in $R_{m}\left(\beta_{r}, 0\right)$, which gives the measure of reduction in the $m$ th Fourier component of the longitudinal potential distribution compared to the infinite stream value. Introducing the normalized potential $\phi$ and current density $\alpha$,

$$
\left.\begin{array}{rl}
\phi(\zeta) & =V(0, \zeta) / V_{0}, \\
\alpha & =\frac{i_{0}}{\frac{-4}{9} \epsilon_{0} \sqrt{-2 \frac{e}{m}} V_{0}^{3 / 2} a^{-2}} \\
& =\frac{i_{0}}{-2.33 \times 10^{-6} V_{0}^{3 / 2} a^{-2}}
\end{array}\right\},
$$

we have two simultaneous single-variable equations to be solved,

$$
\begin{gathered}
\phi(\zeta)=1-\frac{8}{9} \alpha \sum_{m=1}^{\infty}\left[1-\frac{m \pi b}{a} K_{1}\left(\frac{m \pi b}{a}\right)\right] \\
\cdot\left(\frac{1}{(m \pi)^{2}} \int_{0}^{1} \Psi_{z}\left(\zeta^{\prime}\right) \sin \left(m \pi \zeta^{\prime}\right) d \zeta^{\prime}\right) \sin (m \pi \zeta) \\
\Psi_{z}(\zeta)=\phi^{-1 / 2}(\zeta) .
\end{gathered}
$$

This pair of equations was solved iteratively using a digital computer. As a first guess, to start the iterations, the normalized charge density was assumed constant $\left(\Psi_{z}(\zeta)=1\right)$ and the resulting potential calculated from (19). This potential was used to determine a new charge density distribution from (20), and this charge density was in turn used to calculate a new potential from (19), and so on, converging to a sufficiently accurate solution for the potential at the particular value of current; $\alpha$. The largest value of $\alpha$ for which convergence can be obtained is thus the limiting current. This maximum value was determined by increasing $\alpha$ after each convergence (using the solution for the previous value as the new initial trial to reduce computer running time) until a value was finally reached that caused the iterations to diverge. The interval in $\alpha$ between the values giving divergence and the last convergent solution was then successively halved until a sufficiently accurate value for this maximum $\alpha$ was obtained. The numerical solution for potential was allowed to converge to a maximum difference between successive iterations of 0.0001 ; the limiting current was determined within an error $\Delta \alpha$ of $2^{-6}$. The value of $\alpha=8$ was obtained with this accuracy when this method was applied to the infinite stream case, agreeing with the well-known analytic solution [1]-[3]. Eleven terms were taken in the series (19). The values of limiting current, normalized to the infinite stream value, $\alpha=8$, for different values of $b / a$, are shown as open circles in Fig. 2.

An interesting and compact approximate solution can be found for (19) and (20) if two more assumptions are made. First, the space charge is assumed to be distributed axially as $\sin (\pi \xi)$; that is, only the first, $m=1$, term of the series in (19) is retained. Second, limiting is assumed to take place at the same minimum potential as in the infinite diode, $\phi_{m}=\frac{1}{4}$. The increase in limiting current is then simply

$$
\alpha_{\max } / 8=\left[1-\frac{\pi b}{a} K_{1}\left(\frac{\pi b}{a}\right)\right]^{-1} .
$$

This expression is shown as the solid line in Fig. 2, and is seen to be sufficiently accurate for normal design purposes. The potential profiles compared in Fig. 3 indicate that the foregoing assumptions are not unreasonable as long as $b / a$ is not too small. The perveance corresponding to (21) is given in Fig. 4, compared with the 11-term series calculation. Perveance is given by $P=I_{0} /\left(V_{0}\right)^{3 / 2}$, where $I_{0}=\pi b^{2} i_{0}$ for uniform current density. In the limit of small $b / a$,

$$
\underset{b / a \rightarrow 0}{P}=\frac{-11.9}{\ln \left(\frac{\pi b}{2 a}\right)},
$$

which agrees almost exactly with the one-term approximation for $b / a<0.1$; this approximation does not approach a minimum perveance, although the series might have, if carried to smaller $b / a$.

2) Nonuniform Current: Thin Hollow Streams: A simple model of a nonuniform current distribution is now solved. A uniform density of $1+\Delta$ is assumed for $0<r<c$, a density of $p$ for $c<r<b$, and a density of zero for $r>b$. In order to compare correctly the effect of the ununiformity, the same total current is required for this case as for that of the uniform distribution:

$$
\pi b^{2}=\pi(1+\Delta) c^{2}+\pi p\left(b^{2}-c^{2}\right),
$$

which requires, with the definition $c / b=\lambda$,

$$
p=1-\frac{\lambda \Delta}{1-\lambda^{2}} .
$$

The nonuniform distribution to be compared to the uniform-current case is then

$$
\Psi_{r}(\sigma)= \begin{cases}1+\Delta & 0<\sigma<\lambda \\ 1-\frac{\Delta \lambda^{2}}{1-\lambda^{2}} & \lambda<\sigma<1 \\ 0 & \sigma>1 .\end{cases}
$$

The range of the parameter $\Delta$ is

$$
-1<\Delta<\frac{1-\lambda^{2}}{\lambda^{2}}
$$




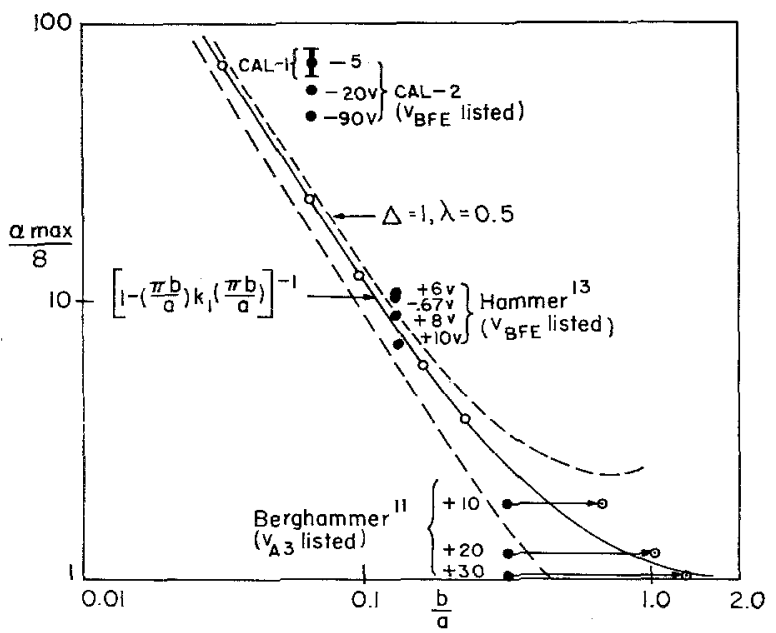

Fig. 2. Increase in maximum value of stable current density of a cylindrical stream as a function of stream aspect ratio $b / a$. The open circles are the series results; the solid line is the first series term, all for uniform current density. The dashed lines are for nonuniform current densities, with $\lambda, \Delta$ defined in Fig. 5 . The solid points are experimental results, as discussed in the text.

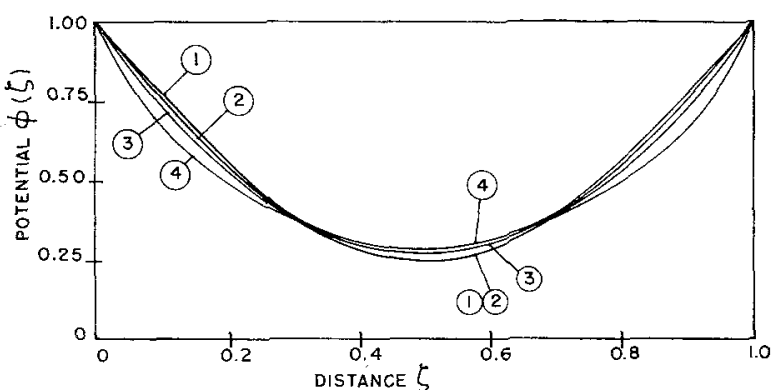

Fig. 3. Potential profiles in the interelectrode space for 1) first term approximation $[1-0.75 \sin (\pi \zeta)] ; 2)$ exact solution for the infinite stream with $\alpha=8 ; 3$ ) computer solution for cylindrical stream with $b / a=0.222816$ and $\alpha=30.50$ (maximum value); 4) computer solution for a cylindrical stream with $b / a=0.0636609$ and $\alpha=182.777$ (maximum value).

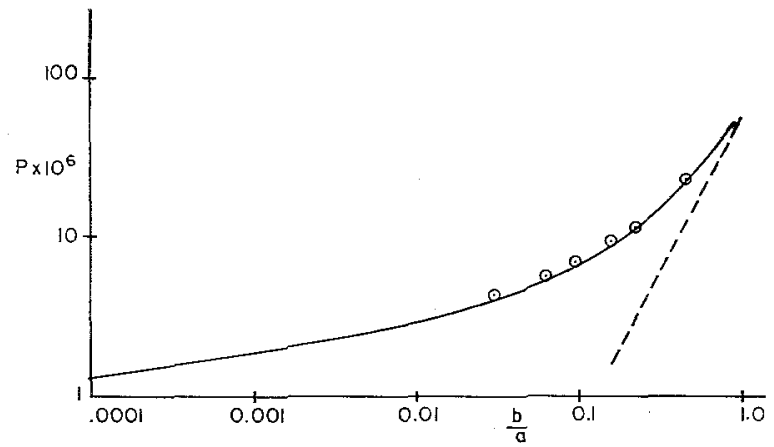

Fig. 4. Limiting perveance, $P=I_{0} / V_{0^{3 / 2}}, I_{0}=\pi b^{3} i_{0}$, for a cylindrical stream of uniform current density. The open cireles are the series results; the solid line is the first series term, which is of questionable value for $b / a \rightarrow 0$. The dashed line, $\sim(b / a)^{2}$, is the asymptote for large $b / a$, the infinite diode.

with these extreme cases illustrated in Fig. 5. The factor $R_{m}\left(\beta_{r}, \sigma\right)$ for this distribution is

$$
\begin{aligned}
R_{m}\left(\beta_{r}, \sigma\right)=\left[1-\beta_{r} I_{0}\left(\beta_{r} \sigma\right) K_{1}\left(\beta_{r}\right)\right] \\
\quad \cdot\left[1+\frac{\Delta}{1-\lambda^{2}}\left\{\frac{1-\lambda \beta_{r} I_{0}\left(\beta_{r} \sigma\right) K_{1}\left(\beta_{r} \lambda\right)}{1-\beta_{r} I_{0}\left(\beta_{r} \sigma\right) K_{1}\left(\beta_{r}\right)}-\lambda^{2}\right\}\right] .
\end{aligned}
$$

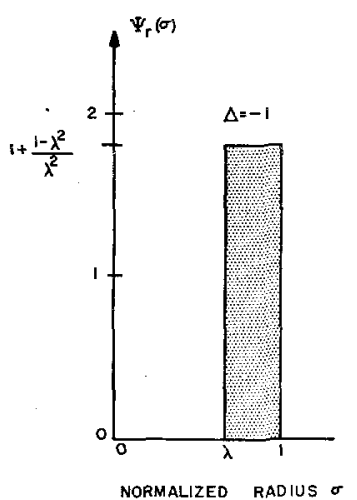

(a)

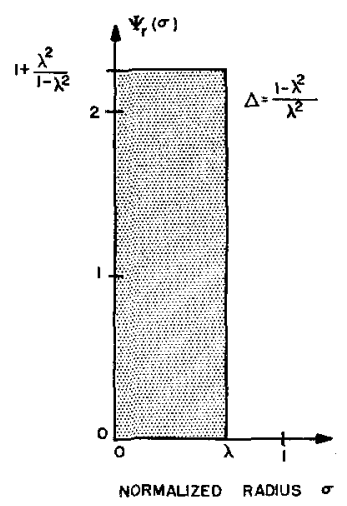

(b)
Fig. 5. Extreme limits of nonuniform transverse current distributions. (a) Hollow stream. (b) Solid cylindrical stream with smaller radius. Distributions (a) and (b) are shown for the same total current.

No iterative computations were made for this type of distribution; the same simplifing assumptions as applied to the uniform stream, however, yield an expression for the approximate increase in limiting current:

$$
\begin{aligned}
& \frac{\alpha_{\max }}{8}=\frac{1}{1-\frac{\pi b}{a} K_{1}\left(\frac{\pi b}{a}\right)} \\
& \cdot\left[1+\frac{\Delta}{1+\left(\frac{c}{b}\right)^{2}} \cdot\left\{\frac{1-(\pi c / a) K_{1}(\pi c / a)}{1-(\pi b / a) K_{1}(\pi b / a)}-\left(\frac{c}{b}\right)^{2}\right\}\right]^{-1} .
\end{aligned}
$$

Equation (28) with $c / b=0.5$ is shown as the two dashed lines in Fig. 2 for the two limiting cases of inequality (26). This approximate expression breaks down for $\Delta=-1$ and large $b / a$, as the potential on the axis has been used to obtain (28), while $\Delta=-1$ defines a hollow stream, and large $b / a$ removes this stream far from the axis. A better answer in this case of a hollow stream would be obtained if the potential $\phi(\sigma, \zeta)$ were evaluated at a point within the hollow stream, say $r=\frac{1}{2}(c+b)$. The other limiting case of (28) is also a bit misleading, since $\Delta=\left[\left(1-\lambda^{2}\right) /\left(\lambda^{2}\right)\right]$ eliminates the stream for $\sigma>\lambda$, defining a uniform stream of diameter $\lambda b$. For this new stream, with a smaller diameter-to-length ratio, the limiting current density is expected to increase rather than decrease as shown; the difficulty lies in the requirement that both streams carry the same total current in an area $\pi b^{2}$. Despite these difficulties, Fig. 2 does show the direction of the change in the limiting current for streams with some nonuniformity, and it demonstrates that in thin streams the correction due to nonuniformity is small.

3) Rectangular Strip Beam: The same method of approximate solution as that applied in the foregoing paragraphs can be applied to a strip beam of thickness $2 h$ and length $a$, illustrated in Fig. 6. The stream is uniform (implying infinite) in the $y$ direction. In this case, a Fourier series-Fourier integral expansion is used. The 


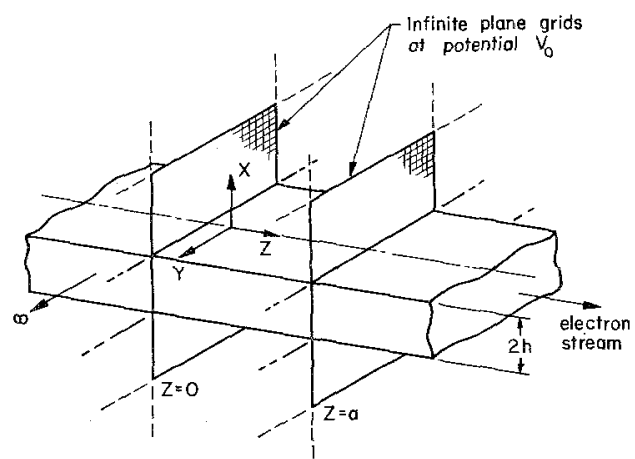

Fig. 6. Stream and electrode configuration for the finite-thickness strip stream model.

resulting equation for potential is the same as (13) with the first term in parentheses, now $R_{m}\left(\beta_{x}, \xi\right)$, given by

$$
\begin{aligned}
& R_{m}\left(\beta_{x}, \xi\right) \\
& \quad=\frac{2}{\pi} \beta_{x}^{2} \int_{0}^{\infty} \int_{0}^{\infty} \frac{\Psi_{x}\left(\xi^{\prime}\right) \cos \left(\gamma_{x} \xi^{\prime}\right) \cos \left(\gamma_{x} \xi\right) d \xi^{\prime} d \gamma_{x}}{\beta_{x}^{2}+\gamma_{x}^{2}}
\end{aligned}
$$

The normalization is

$$
\begin{aligned}
\xi & =x / h, \\
\beta_{x} & =m \pi h / a, \\
\gamma_{x} & =k h .
\end{aligned}
$$

With a uniform current density (29) becomes

$$
R_{m}\left(\beta_{x}, \xi\right)=1-\exp \left(-\beta_{x}\right) \cosh \left(\beta_{x} \xi\right)
$$

for $\xi<1$. The same assumptions used with the cylindrical stream are applied here to obtain the approximate limiting current density :

$$
\begin{aligned}
\frac{\alpha_{\max }}{8} & \cong\left[1-\exp \left(\frac{-\pi h}{a}\right)\right]^{-1} \\
& =\frac{a}{\pi h} \text { for } h \rightarrow 0 .
\end{aligned}
$$

This is shown as the solid line in Fig. 7. Numerical solutions for the equations corresponding to (19) and (20) are again shown as open circles. Equation (31) is obviously sufficient for design purposes. The perveance corresponding to (31) is given in Fig. 8, compared with the series calculation; there is an apparent asymptote for $h / a \rightarrow 0$. The maximum current is given by $\left(i_{\max }\right)$ (area); let the cross section be $(a)(2 h)$, so that

$$
\begin{aligned}
P_{\max } & =\frac{I_{\max }}{V_{0}^{3 / 2}}=\frac{16 \cdot 2.33}{10^{6}} \frac{\alpha_{\max }}{8} \frac{h}{a} \\
& =11.85 \text { for } h \rightarrow 0 .
\end{aligned}
$$

A nonuniform, triangular distribution is also treated [14]. The extreme cases of this distribution are shown dotted in Fig. 7; the effect is obviously small. A Gaussian distribution has also been treated by one of the authors ${ }^{3}$ with results very close to those for the uniform stream.

${ }^{3}$ Bridges, W. B., unpublished work.

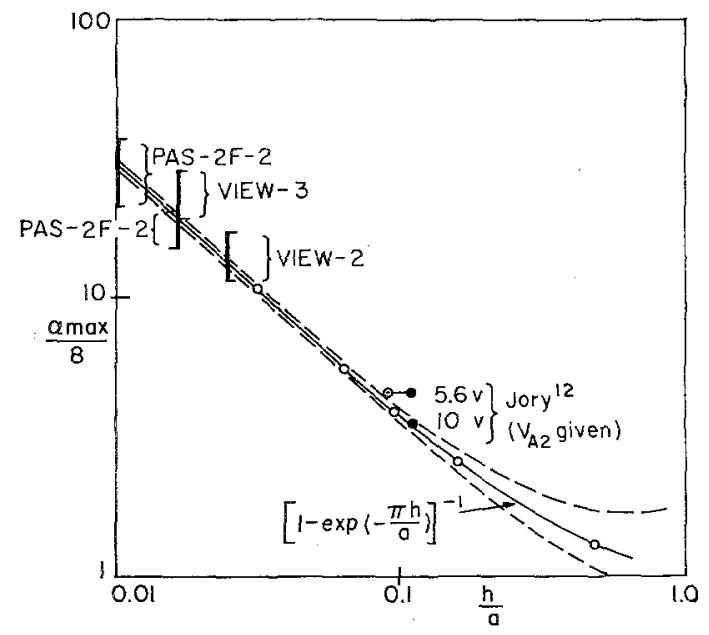

Fig. 7. Increase in maximum value of stable current density of a strip stream as a function of stream aspect ratio $h / a$. The open circles are the total series calculations; the solid line is the first series term, all for uniform current density. The dashed lines are for triangular density variations, with current concentrated near the stream edge (upper line) and near the axis (lower line). The experimental points are discussed in the text.

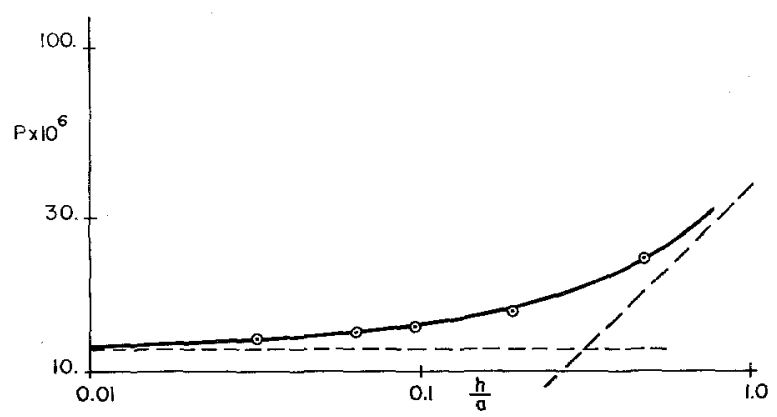

Fig. 8. Limiting perveance, $P=I_{0} / V_{0}^{3 / 2}, I_{0}=i_{0} 2 h a$, for a strip stream of uniform current density (perveance for a width $2 a$ along $y$ ). The open circles are the series results; the solid line is the first series term, which has a limiting value of 11.85 for $h / a \rightarrow 0$. The sloping dashed line, $\sim h / a$, is the asymptote for large $h / a$, the infinite diode.

\section{Experimental Results}

A number of experiments [11]-[14] illustrate the validity of the forgoing results. The observed values of increase in maximum stable current are given on Figs. 2 and 7 for different beam aspect ratios, $b / a$ and $h / a$.

A typical electrode arrangement (CAL-2) is shown in Fig. 9. The current-limiting region lies between anodes 3 and 4 (the ends of a cylindrical cavity in this tube); anodes 2 and 5 are guard anodes, held at a slightly higher potential than anodes 3 and 4 to retard secondary emission and to shield the beam apertures in anodes 3 and 4 from the strong fields produced by the potentials on anode 1 and the collector.

For the cylindrical-stream experiments (Fig. 2) the observed values of the limiting-current increase are seen to be generally higher than the simple theory predicts by as much as three times. Partial neutralization of the beam space charge by the residual ions could account for part of this increase. The potential depression formed in the beam near limiting strongly attracts any ions 


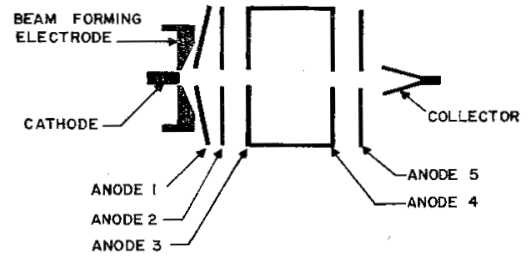

Fig. 9. Typical electrode arrangement (CAL-2) used in currentlimiting experiments.

formed and traps them. Unfortunately, pulsed experiments which might have resolved the question of ion neutralization were not performed, although it is likely that pulsed currents would cause "premature" limiting because of the lack of equilibrium conditions. Such effects are discussed by Bridges and Birdsall [9].

The points shown for CAL-2 (Fig. 2) are for different values of beam-forming-electrode (BFE) potentials with $V_{0}$ fixed. Limiting occurred at lower currents for the more negative BFE potentials. This is in agreement with the result obtained here for the nonuniform radial distribution of current; negative BFE potentials tend to inhibit emission from the cathode edges and hence produce a beam of smaller diameter, giving a distribution similar to the nonuniform beam model with $\Delta=+3$.

The four points shown for Hammer's experiment [13] are also for different $\mathrm{BFE}$ potentials, but the variation is in the opposite direction from that expected from the foregoing discussion. Because limiting occurred between anodes 2 and 3 (no input guard anode), field penetration from anode 1 may have more than offset the change in the transverse distribution of current, since the potential of anode 1 was increased as the BFE potential was decreased.

Only diodes symmetrical about $z=a / 2$ have been treated here, although it is clear how to apply the present computational method or obtain the analogous simplified expression for asymmetrical cases. Limiting in asymmetrical diodes, such as those in Berghammer's experiment [11], may be compared, however, through the following argument. In Berghammer's tube, limiting was obtained between anodes 2 and 3 , with anode 2 at 2 volts and anode 3 at 10,20 , and 30 volts for the three points shown. As the anode 3 potential increases, the potential minimum moves closer to anode 2 (the input electrode). The electric field and charge distribution between the input electrode and the potential minimum are similar to those in a symmetrical diode with spacing $2 z_{m}$, where $z_{m}$ is the distance from the injection plane to the potential minimum, instead of $a$. If the observed values of limiting-current increase are associated with aspect ratios $b / 2 z_{m}$, rather than $b / a$, the points move to the right (as shown by arrows) and the agreement with the theoretical curve of Fig. 2 is improved.

Figure 7 shows the experimental results for tubes using thin hollow streams. All tubes used had a stream diameterthickness ratio of more than ten, so that the approximation to a rectangular strip beam is quite good. Jory's tube [12] and the PAS-2F-2 were both experimental low-noise back- ward-wave amplifiers with multianode guns. Tubes VIEW2 and VIEW-3 were beam testers using guns similar to that in PAS-2F-2. The range shown for each tube corresponds to different values of limiting-region potential. Again, a change in gun optics is probably the cause for the spread. Of the two points taken from Jory, the lower was obtained with a symmetrical limiting region and the upper with an asymmetrical region. If $h / 2 z_{m}$ is used as the applicable aspect ratio instead of $h / a$, the upper point moves to the left, as shown by the arrow.

\section{Strream in a Finite-Length Drift Tube ${ }^{4}$}

The model treated in Section II is a sheet stream as shown in Fig. 6, but with an additional set of parallel planes above and below the stream added to form a closed box, as illustrated in Fig. 10.

\section{A. Method of Solution}

The basic equations to be solved are as before, written now in the rectangular coordinates $x$ and $z$. Approximate solutions are again found to be necessary. The technique analogous to that used in Section I would be the expansion of potential and charge density in a double Fourier series. This technique was initially used to solve certain cases of the gridded drift tube problem, but the two-dimensional array of coefficients required to obtain a sufficiently accurate answer resulted in unreasonably large computer solution time. Instead, a relaxation technique was adopted which greatly shortened the computation time.

Introducing the normalization

$$
\begin{aligned}
& X=x / D, \\
& Z=z / L, \\
& \phi=V / V_{0}, \\
& A=i_{0} L^{2} /-2.33 \cdot 10^{-6} V_{0}^{3 / 2},
\end{aligned}
$$

(1) becomes

$$
\left(\frac{L}{D}\right)^{2} \frac{\partial^{2} \phi}{\partial X^{2}}+\frac{\partial^{2} \phi}{\partial Z^{2}}=\frac{-\rho L^{2}}{\epsilon_{0} V_{0}} .
$$

The equations of motion (2), (3) give the expression for charge density $\rho$ :

$$
\rho=\frac{-4}{9} \frac{A \epsilon_{0} V_{0}}{\phi^{1 / 2} L^{2}}
$$

Using a digital computer, (34) and (35) were solved iteratively, subject to the boundary conditions imposed by the conducting walls. At each step in the iteration, (34) was solved by the method of successive over-relaxation, using the charge density at that step. In this method, described in detail by Hornsby [15], an accelerating factor, which adjusts the amount of over-relaxation, was used to reduce the computing time. A special method for

\footnotetext{
${ }^{4}$ Section II describes work performed in partial satisfaction of the requirements for the M.S. degree of J. I. Frey, University of California, Berkeley.
} 


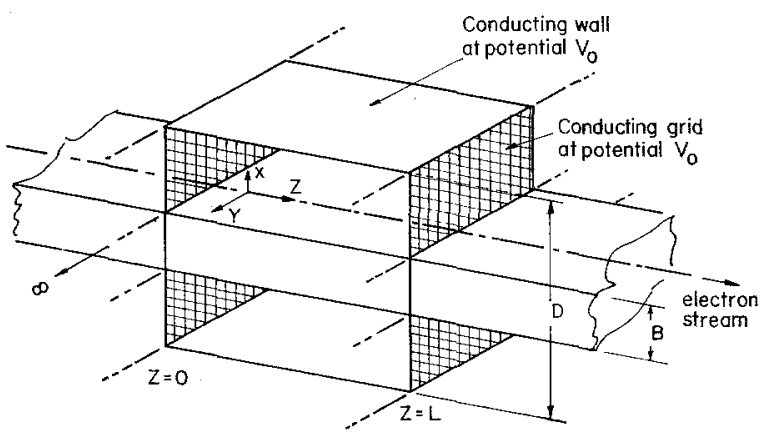

Fig. 10. Stream and electrode configuration for the finite-length drift tube model.

choosing optimum values of this factor, described by Carré [16], was used. As a consequence, the bulk of the computing time was consumed by the major iteration loop (charge density to potential and back again).

The numerical solution for potential was allowed to converge to a maximum difference between successive iterations of 0.00005 (normalized). For this problem, it was discovered that larger differences masked situations of marginal convergence very near limiting; instead of converging to a final answer, the potential would first converge and then diverge very slowly. Because of this difficulty and the necessity of performing the computations for many sets of the parameters $L / D$ and $B / D$, "limiting" was taken as the point where the slope of the minimum potential vs. input current curve exceeded ten times its value at zero current.

The fineness of the mesh used for the relaxation solution was tested by increasing the number of points at which potential was calculated from 441 to 1641 . Since the maximum difference in results produced by this change was less than 0.001 per cent, all calculations were performed with the 441-point mesh. As an overall check of the computation, current dependence of the potential in an extremely short diode $(L / D=0.05, B / D=1.0)$ was calculated. The curve of potential mimimum vs. input current agreed with that for an infinite diode within 0.01 per cent up to $A=6$ (the infinite diode limits at $A=8$ ); beyond this value, the effect of the side walls began to be noticeable.

It was possible to use any spatial distribution of potential as an initial estimate for each computation. Most calculations were started at low input currents with a uniform-potential estimate; the solution obtained was then used as a first estimate for the next, increased-current calculation. To ascertain whether or not there might be another stable potential distribution in addition to the single-minimum solution found by the sequence described, two calculations were started with input currents only slightly below the critical value and with potential distributions having two and three minima. In each case, the several minima in the potential distribution disappeared within two or three iterations and the previously found single-minimum solution was ultimately obtained; this solution is thus assumed to be unique.

\section{B. Computational Results}

All results discussed here were obtained for a uniform current distribution across the beam width $B$ at the input grid of the drift tube. Limiting current as a function of the stream aspect ratio is given in Fig. 11, normalized to a limiting current in an infinitely broad diode. Also shown are the data from Section I for the sheet stream in a diode, (i.e., the side walls have been removed to infinity, $D \gg B$ ). The effect of the tube walls in reducing the space-charge potential depression is apparent; the closer the boundary to the stream edge, (i.e., the lower the value of $D / B$ ), the greater the increase in the limiting current. As the ratio of beam thickness to length is increased (large $B / L$ ) the side walls play a less important role.

In the opposite limit, where $L \gg D, B$, the model approaches that of an infinitely-long drift tube. For design purposes, it would be useful to know the value of $L / D$ above which the result for the infinite drift tube is valid. Haeff [4] has derived the following expression for the limiting perveance of a stream in such a drift tube:

$$
P_{\max }=9.35 \cdot 10^{-6} \frac{w}{D} F_{\max } .
$$

Here, $w$ is the depth in the $y$ direction, and $F_{\max }$ is a generalized current parameter, somewhat like our $A$, but dependent upon the filling factor $w / D$. For the finitelength drift tube, the maximum perveance is given by

$$
P_{\max }=B \cdot W \cdot \frac{2.33 \cdot 10^{-6}}{L^{2}} A_{\max } .
$$

Consequently, the ratio of these perveances for any $B / D$ or $L / D$ is

$$
0.249 \frac{B / D}{(L / D)^{2}} \frac{A_{\max }}{F_{\max }} .
$$

Equation (38) is plotted in Fig. 12 for two values of $B / D$, using $F_{\max }$ from Haeff's paper and $A_{\max }$ from the present numerical calculations. The limiting behavior of the finitelength tube approaches that of the infinite-length tube within five per cent for $L / D>4$ if the stream fills the tube, and $L / D>2.5$ if the stream only fills half of the tube.

Figure 13 shows the effect of length-to-diameter ratio $L / D$ on minimum potential in the tube for the stream filling the tube, $D / B=1$. The fact that the stream is entirely enclosed does not change the shape of these curves from those of the infinite diode or infinitely-long drift tube. However, as the tube is made more "box-like" ( $L / D$ approaching unity), the minimum potential at limiting drops below the value of 0.25 at limiting obtained in the infinite diode and infinitely-long tube (with $B / D=1$ ). 


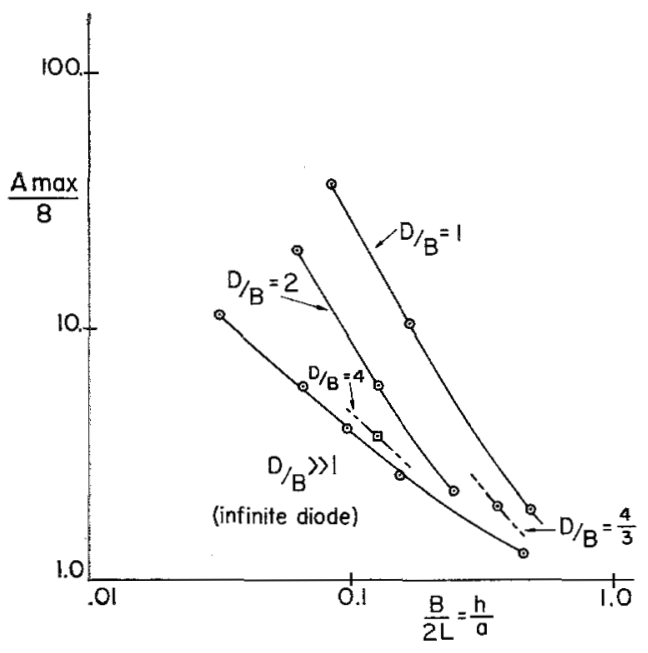

Fig. 11. Increase in maximum stable current density for a strip stream as a function of stream aspect ratio $h / a$ (as in Fig. 7) with addition of side walls, with aspect ratio of $D / B$.

\section{Experiments}

Experimental results for unneutralized electron streams in cylindrical drift tubes with gridded ends have been reported by Atkinson [17]-[18] and Volosok and Chirikov [19]. These experiments were, however, performed in order to study the behavior of streams at and beyond limiting current, independent of longitudinal boundaries. Consequently, the $L / D$ ratios used were generally large enough so that, as predicted by the present work, the behavior duplicated that of the infinitely-long systems. The fact that Atkinson observed no change in behavior for a stream with $B / D=0.134$ when $L / D$ was increased from 1.5 to 3.0 tends to affirm the implications of Fig. 12 .

\section{Calculation of Kinetic and Electric Energies}

The relative magnitudes of the kinetic and electric (or potential) energies in the drift tube can be compared with results obtained by Bridges and Birdsall for the infinite diode [9]. The kinetic energy in the stream is obtained from the integral

$$
\begin{aligned}
W=\iint_{\text {stream }} \frac{1}{2} m v^{2} & \left(-\frac{\rho}{e}\right) d x d z \\
& =\frac{1}{2} i_{0} \iint \sqrt{-2 \frac{e}{m} V(x, z)} d x d z
\end{aligned}
$$

while the electric energy is obtained from

$$
W_{E}=\frac{1}{2} \epsilon_{0} \iint_{\text {tube }}|E|^{2} d x d z
$$

These integrals were calculated numerically. The potential within a mesh square was taken as the average of the potentials at the corners; the electric-field components within a square were considered to be the averages of the field components calculated by subtracting the proper

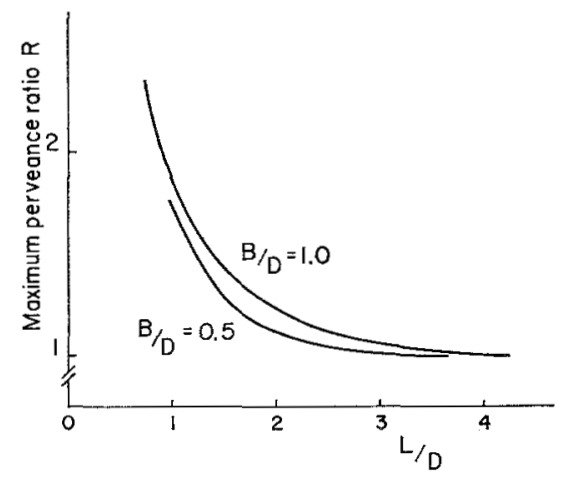

Fig. 12. Ratio of limiting perveance of drift tube of length $L$ to that for $L \rightarrow \infty$ for stream filling and half-filling the cross section.

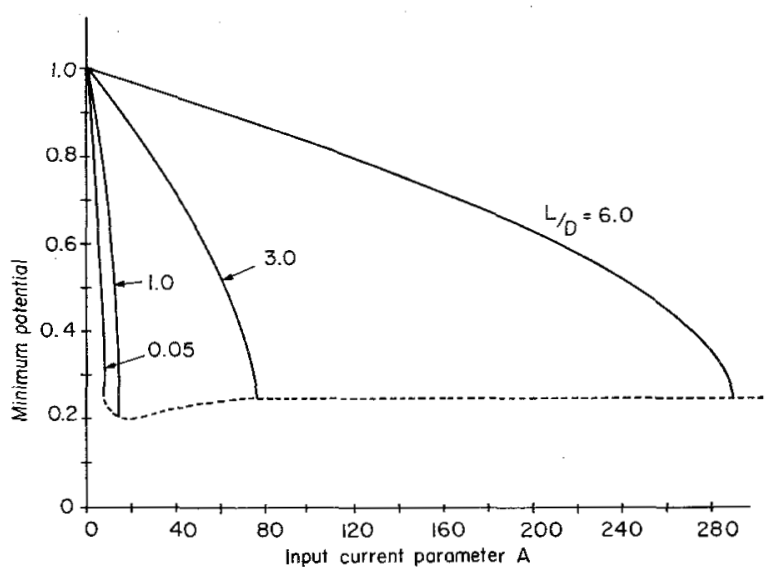

Fig. 13. Minimum potential in drift tube as a function of input current for different $L / D$ ratios, for stream filling the drift tube. $D / B=1$.

corner potentials and dividing by the length of a side of a mesh square.

The result of the energy calculation for the "square" stream $(L / D=1)$ is shown in Fig. 14. $\partial W_{K} / \partial I$ and $\partial W_{E} / \partial I$ are large and of opposite sign at limiting for this case, and $\partial W / \partial I$ is large and positive, indicating instability for currents greater than $I_{\max }$. (It is, of course, impossible to show infinite slopes from numerical results.) Similar behavior is exhibited for all other values of $L / D$ and $B / D$ for which calculations were made, and is very similar to that obtained for the infinite diode [9].

For the interesting limiting case of a very thin stream in a very long drift region, the energies can be calculated in closed form using a method suggested by Nergaard [4]. As shown in Fig. 15, the behavior of the energies as a function of current is quite similar to that demonstrated in the infinite diode. At limiting current, $\partial W_{K} / \partial I, \partial W_{E} / \partial I$, and $\partial W / \partial I$ all become infinite, indicating a rapid onset of instability. However, in the infinite diode, at this point $W_{K}=7 / 4 W_{E}$, while here $W_{K}=W_{E}$. It is proposed that this difference may result in a smaller amplitude of oscillation subsequent to the onset of instability, as noted earlier [9] for the thin beam in a diode, and may also result in a smaller drop in transmitted current. 


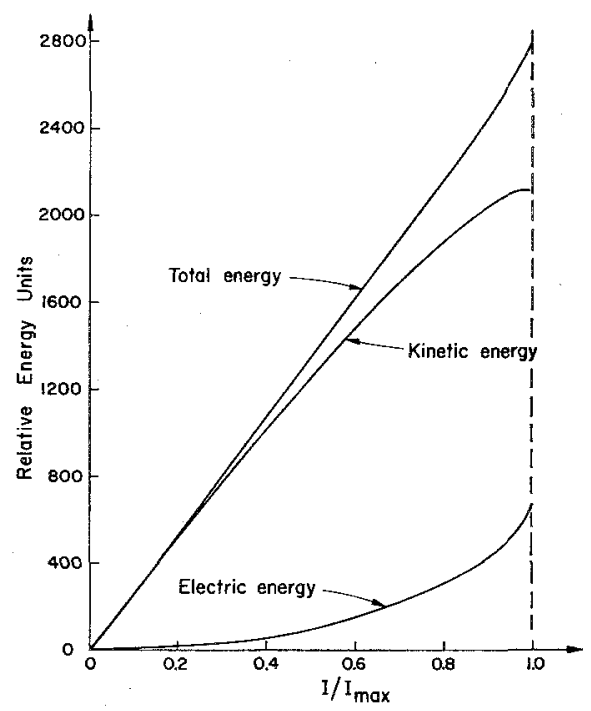

Fig. 14. Energies in a bounded drift tube as a function of input current. $L / D=1, D / B=1$.

\section{ACKNOWLEDGMENT}

The authors are grateful to G. A. Becker at the University of California, Berkeley, and R. B. Hodge at Hughes Research Laboratories, Malibu, Calif., for fabrication of the experimental tubes, and to J. M. Hammer of RCA Laboratories, Princeton, N.J., and H. R. Jory of Varian Assoc., Palo Alto, Calif. for use of their experimental data. We would also like to thank our many colleagues for their discussions and suggestions.

\section{REFERENCES}

[1] Fay, C. E., A. L. Samuel, and W. Shockley, On the theory of space charge between parallel plane electrodes, Bell Sys. Tech. $J .$, vol 17, Jan 1938 , pp 49-79.

[2] Salzburg, B., and A. V. Haeff, Effects of space charge in the grid-anode region of vacuum tubes, $R C A R e v$., vol 2, Jan 1938, pp 336-374.

[3] Strutt, M. J. O., and A. Van der Ziel, On electronic space charge with homogeneous initial electron velocity between plane electrodes, Physica, vol 5, Aug 1938, pp 705-717.

[4] Haeff, A. V., Space charge effects in electron beams, Proc. IRE, vol 27 , Sep 1939, pp 952-971.

[5] Smith, L. P., and P. L. Hartman, The formation and maintenance of electron and ion beams, $J$. Appl. Phys., vol 2, Mar 1940 , pp 220-229.

[6] Pierce, J. R., Theory and Design of Electron Beams. Princeton, N. J.: Van Nostrand, 1949.

[7] Brewer, G. R., Graphs of potential and perveance of an electron beam in a confining magnetic field, ETL Memo 9, Research and Development Labs., Hughes Aircraft Co., Jun 1953, p 11.

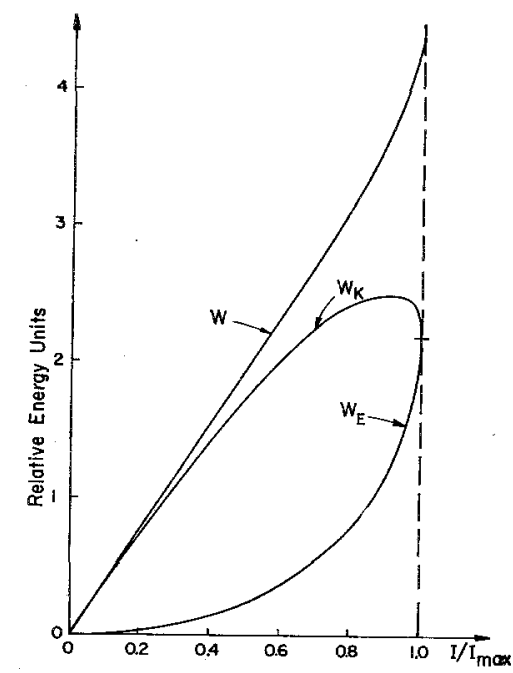

Fig. 15. Energies in a bounded drift tube as a function of input current. $L / D \rightarrow \infty, D / B \rightarrow \infty$.

[8] - Graphs of the space charge depression of potential in a cylindrical electron beam, ETL Memo 55, Research and Development Labs., Hughes Aircraft Co., May 1955, p 26.

[9] Bridges, W. B., and C. K. Birdsall, Space charge instabilities in electron diodes. II, J. Appl. Phys., vol 34, Oct 1963, pp 2946-2955.

[10] Branch, G. M., and T. G. Mihran, Plasma frequency reduction factors in electron beams, IRE Trans. on Electronic Devices. vol ED-2, Apr 1955, pp 3-11.

[11] Berghammer, J., Space charge effects in ultra-low-noise electron guns, RCA Rev, vol 21, Sep 1960, pp 369-376.

[12] Jory, H. R., Noise in backward-wave amplifiers, Ph.D. dissertation, University of California, Berkeley, 1960.

[13] Bloom, S., J. Berghammer, A. L. Eichenbaum, and J. M. Hammer, Low-noise microwave tubes, First Semi-Annual Rept, RCA Labs., Princeton, N. J., Mar 1961. (Contract DA-36-039 SC 87393).

[14] Bridges, W. B., and C. K. Birdsall, An electron stream instability, Rept 443, Electronics Research Lab., University of California, Berkeley, Mar 1962. (Available from DDC, No. AD 284291)

[15] Hornsby, J. S., A computer program for the solution of elliptic partial differential equations (potential problems) C.E.R.N. Rept 63-7, Mar 26, 1963.

[16] Carre, B. A., The determination of the optimum accelerating factor for successive over-relaxation, Comput. $J$., vol 4, Apr 1961, pp 73-78.

[17] Atkinson, H. H., Limiting eurrents and neutralization in cylindrical electron beams, presented at Fourth International Congress on Microwave Tubes, Scheveningen, Holland, Sep 3-7, 1962 .

[18] _... Limiting perveance of electron beams with and without positive ions, presented at Conference on Instabilities due to Streams or Currents, Berkeley, Calif., Apr 7, 1964.

[19] Volosok, V. I., and B. V. Chirikov, Space charge compensation in an electron beam, Soviet Phys.-Tech. Phys., vol 2, Nov 1957, pp 2437-2442. 